\title{
SUMMARY OF RECENT TARGET STUDIES
}

\author{
F. Bieniosek and S. O'Day
}

4-Feb-1993

This report describes recent measurements that have been performed with the new target stack (Fig. 1). Highlights of these measurements are listed below.

1. Pbar yields of nickel and powdered rhenium are comparable to that of copper.

2. Enhancement of pbar yield at the interface between copper and aluminum disks in the target stack has been observed. This effect occurs only when the lens is focused near the upstream edge of the target.

3. The target density depletion study in powdered rhenium showed an apparent yield reduction on the time scale of a single proton pulse, accompanied by release of airborne radioactive material.

Yield comparison of target materials. Table 1 shows a series of measurements using P87 histograms of various ratios relevant to target performance. Representative measurements of pbar yields (normalized) are listed in Table 1. The materials are copper, nickel, and powdered rhenium compressed to $75 \%$ solid density inside a titanium can. The D:YIELDI measurements were performed with $1.2 \times 10^{12}$ protons on target; the D:FFTTOT measurments were performed with $1.6 \times 10^{12}$ protons on target. Little difference in target yield was observed for the three materials. Recently the target has been operating on nickel. Nickel was chosen, first, because it generally shows slightly higher yields than copper (although the improvement is probably not statistically significant), and second, to obtain operating experience with this metal, which should be capable of operating at higher energy density on target than copper.

Table 1

material

D:YIELDI

M:TOR109

D:FFTTOT

M:TOR109

$\mathrm{Ni}$

1.000

1.000

$\mathrm{Cu}$

0.979

0.997

$\mathrm{Re}$

0.994

0.998 
Edge enhancement of vield. This study was designed to test a prediction of enhanced yield at the interface between copper and aluminum, under the appropriate conditions. The enhancement is due to the reduced attenuation of antiprotons created in the dense material (copper), but exiting the target through the light material (aluminum). Normally, the target is positioned with its center near the focal point of the lithium lens. No edge effect has been observed in this case. However, if the target is moved such that the lens is focused on the upstream end of the target, the total pbar yield drops by about $30 \%$, but the differential edge effects are more easily observed because of the longer path lengths of the antiprotons through the dissimilar materials. Figure 2 shows the yield to IC728 as a function of vertical position of the target (D:TRY) at the interface between a copper cooling disk and the bottom of the aluminum target. The lens is at its normal position (D:TRZ $=19.5 \mathrm{~cm}$ ). The edge effect is not observed. Figure 3 shows the yield at the top edge of the aluminum target, with $D: T R Z=15.43 \mathrm{~cm}$, i.e. the lens is focused at the upstream edge of the target. Larger values of D:TRY correspond to the titanium can of the powdered rhenium target, and smaller values correspond to the aluminum target disk. In this case the edge effect (an enhancement in yield at the edge of the copper portions, and a reduction at the edges of the aluminum disk) is clearly observed. Figure 4 shows a similar scan of an identical cooling disk located between a copper and a nickel disk. The edge effect is gone, (lower trace, $D: T R Z=15.43$ ) except perhaps for a small effect at the interface at the copper/air cooling channel. The upper trace is the yield with the target at its normal position. Again, no edge effect is observed. (Note the hysteresis in the readback D:TRY.)

Detailed measurements at the upper and lower interfaces were performed with P87 to verify the effects on the pbar yield D:YIELDI. These results are summarized in figure 5. Again, there is an enhancement at the edge of copper, and a reduction at the edge of aluminum. The measured effect is $4-5 \%$ in amplitude in both cases. The result was expected, and is due to changes in attenuation of the pbars as they leave the target.

The demonstration of edge enhancement indicates that it should be possible to increase yield by designing a target that takes advantage of the edge effect, specifically, a wire target with about a 1-mm diameter. Detailed modelling of pbar production, including realistic estimates of the rate of secondary production of pbars, will be necessary to determine the expected amount of yield increase.

Density depletion study. Large energy density on target is expected to melt target materials and hence cause density depletion, accompanied by a reduction in yield. The potential exists for target melting to occur routinely under Main Injector conditions, in the absence of a beam-sweeping system. Therefore it is important to understand the behavior of the target under the extreme conditions expected with high-intensity Main Injector beams. Since rhenium has a relatively low melting-point energy, we performed a study to demonstrate density depletion in rhenium. In this study the beam 
intensity on target (M:TOR109) was about $1.6 \times 10^{12}$ protons per pulse, and the beam was tightly focused $(\sigma \simeq .14 \mathrm{~mm})$. The debuncher gap monitor signal on the first turn was measured and integrated for several bunches throughout the 82-bunch beam pulse. Fig. 6 shows the integrated signal as a function of bunch number. The points marked 'first' and 'last' were the first and last data points taken in the sequence, and thus represent the level of reproducibility of the data. Note the reduced signal in the late bunches of the beam pulse. This reduction, when normalized against the incoming bunch structure (see below), indicates a yield reduction in the range of $8 \pm 4 \%$ over the final portion of the pulse.

The proton bunch structure was measured by integrating the current in proton bunches passing the AP1 wall current monitor, upstream of the target. The data, shown in Fig. 7, indicate a downward slope in the signal as a function of bunch number. The final bunches are about $8 \%$ smaller than the initial bunches. The droop is attibuted to beam loading in the main ring. Finally, data taken in a nickel target under conditions similar to those in the rhenium target investiation (Fig. 8) show no drop in yield toward the end of the pulse. This result indicates that no density depletion is taking place in nickel, as expected.

\section{Discussion of the density depletion study.}

During the course of the investigation with rhenium, the airborne radiation monitor in the building APO showed a rapidly rising count rate. The experiment was halted after 2 hours, and the count rate peaked at approximately 10 times the normal count rate. Subsequent analysis showed the presence of radionuclides (predominantly iodine, with smaller amounts of tellurium, sodium, and potassium) in the airborne radiation monitor [Ref. 1]. The total release was relatively small (about $5 \mathrm{mCi}$ ). A likely source for these particles is the following. It is known that bombardment of metals by a high-energy proton beam produces noble gases by spallation reactions between the target nuclei and the incident protons [Ref. 2]. This fact is of general interest in determining the rate of void formation, swelling, and radiation embrittlement of solid target materials. In the case of rhenium, the predominant gas is xenon. The numbers in the CERN paper would indicate a production on the order of $10^{12}$ xenon atoms in the course of our experiment. We hypothesize that the xenon, normally trapped in the solid metal target, was released as the target material melted, and subsequently escaped through the voids in the pressed-powder target material and the seams of the titanium can. Radioactive xenon nuclei would then have decayed to the daughter products observed (iodine and tellurium). A similar, although much smaller, release occurred in the earlier study at $1.2 \times 10^{12}$ protons.

One would expect similar release of radioactive argon and daughter products in the case of melting solid copper and nickel targets under Main Injector conditions. However, the amount of release may be smaller because of the smaller surface area available to the escaping gases in a solid target. A beam-sweeping system that 
reduces peak heat load on target, and a ventilation system that blows air down into the prevault/tunnel area, will help prevent future releases of such gases.

Although the quality of the data is relatively poor, and information on the detailed thermodynamic properties of rhenium is not always available, it is important to try to develop a comparison between the measured yield reduction and expected density depletion in the powdered rhenium. The simple model of Ref. 3 may be used to estimate the temperature of the material implied by the measured amount of reduction in yield. The model assumes that the density reduction is the result of the formation of a channel of liquid metal in the target material. Typically the dependence of density of liquid metals on temperature takes the form

$$
\mathrm{d} \rho / \mathrm{dT}=-\mathrm{K} \rho
$$

where the constant $K$ is in the range 0.5 to $2 \times 10^{-4}$. No data exist for the density variation of liquid rhenium with temperature, but the central value in the range $\left(1 \times 10^{-4}\right)$ corresponds to copper. Calculations have been made for this value of the constant $K$ [Ref. 3]. If we use this value of $\mathrm{K}$, a yield reduction of $8 \%$ implies a central density reduction of $16 \%$ in a thin target. Thick-target effects modify this result slightly. For example, if the absorption of the incident proton beam is $50 \%$ through the entire target, absorption to the center of the target is about $25 \%$. Taking this into account, the indicated density reduction is about $\Delta \rho / \rho=-21 \%$. A density reduction of this magnitude corresponds to a temperature rise beyond the melting point of $2100^{\circ} \mathrm{C}$ (from eq. 1). Since the melting point of $R e$ is $3180^{\circ} \mathrm{C}$, this result implies melting begins about $60 \%$ into the pulse. The final temperature is $5280^{\circ} \mathrm{C}$.

From another point of view, energy deposition in rhenium for $1.6 \mathrm{x}$ $10^{12}$ protons should be about $1070 \mathrm{~J} / \mathrm{g}$ for $\mathrm{a} \sigma=0.14 \mathrm{~mm}$ beam spot. The melting point energy for rhenium (about $613 \mathrm{~J} / \mathrm{g}$ ) then indicates melting begins about $57 \%$ of the way into the pulse. The agreement between the two calculations, while certainly not conclusive, suggests that the target material behaved about as one would expect.

Finally, it is interesting to note that, even with energy deposition far exceeding the theoretical limit for melting the target, the reduction in total yield was not significant (Table I). One can conclude that the practical limit to target energy density due to short-term density depletion is significantly higher than the melting point of the material. Other limiting effects, such as shock-wave damage, long-term radiation damage, and especially production of airborne radionuclides, may ultimately play a more significant role in limiting permissable energy density on target.

\section{References}


1. T. Leveling, "Estimate of Activity Released During Use of Rhenium Target 1/13/93."

2. T. W. Eaton, C. D. Johnson, E. Jones, CERN/PS/868-15 (May 1986).

3. F. M. Bieniosek, Pbar note \#512, 10 Sep 1991. 

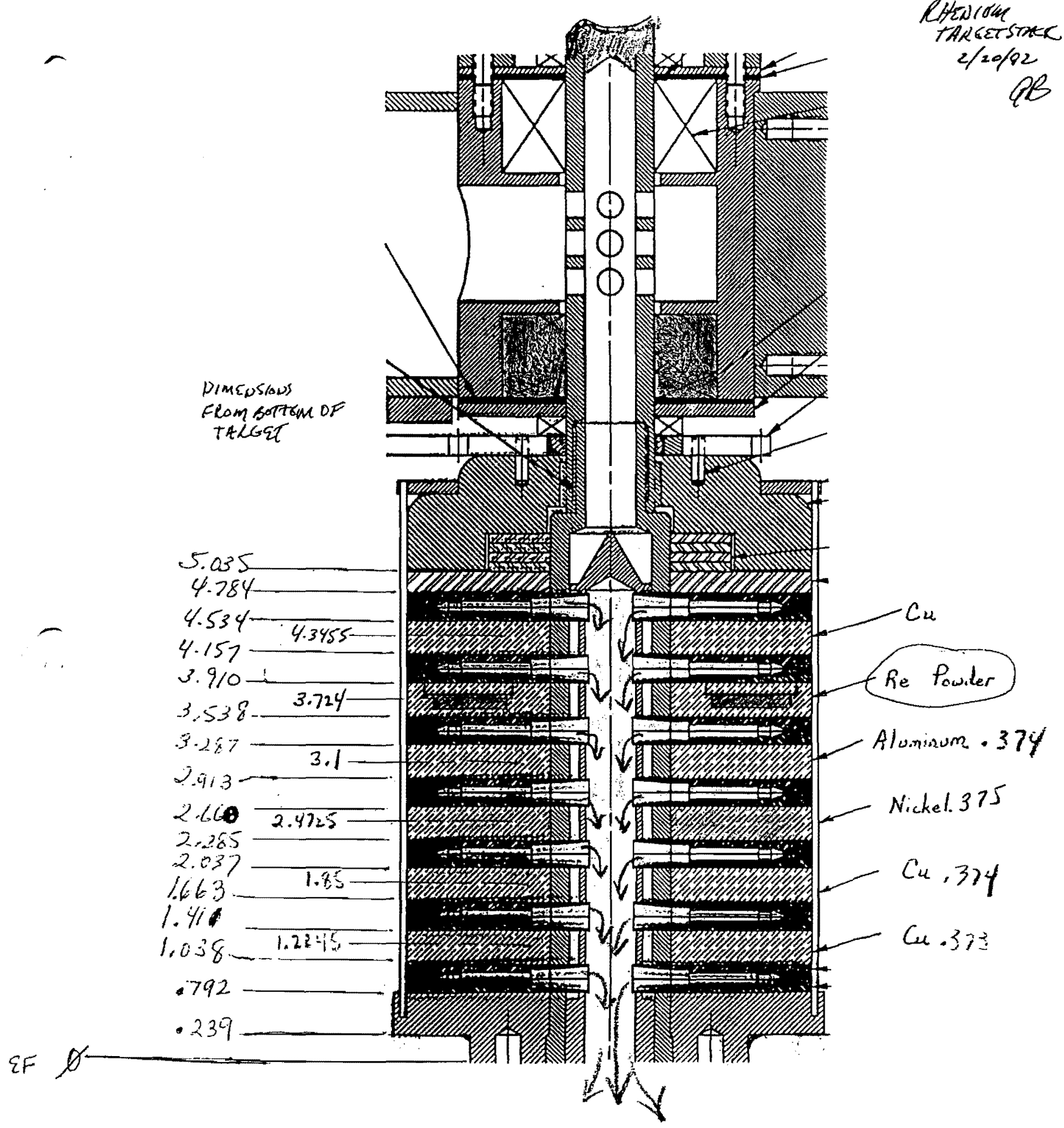

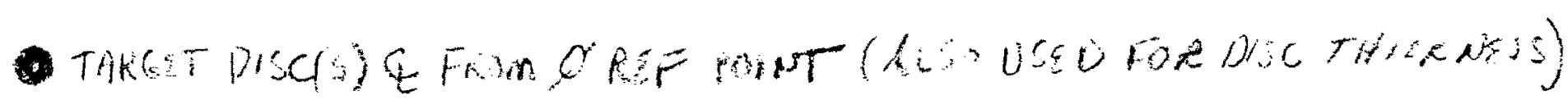

FIGURE 1. 


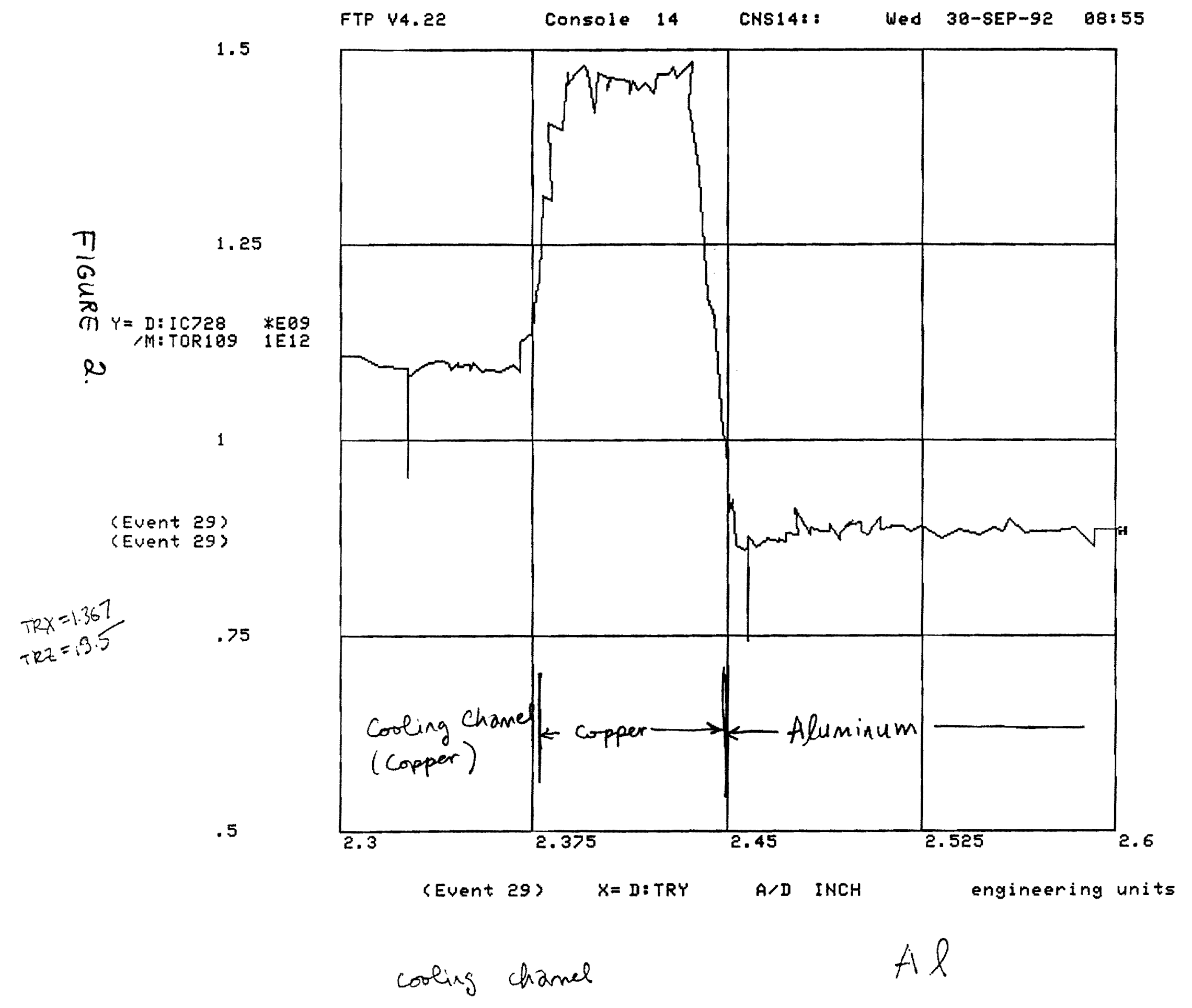

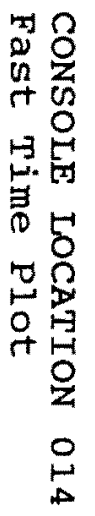

$\omega$
0
1
0
$\pi$
0
1
0
0
0
$N$
0
0
0
0

corling chamel 


$$
\begin{aligned}
& R x=1.3 \\
& 2 x=5+3
\end{aligned}
$$

1.25

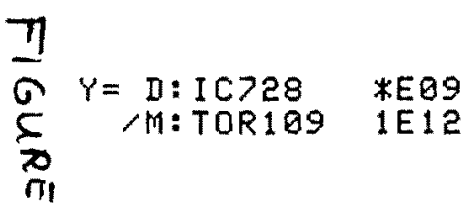

$\omega$

$$
\begin{aligned}
& \text { (Event 29) } \\
& \text { (Event 29) }
\end{aligned}
$$

.75

.5
Console 14 CNS14:2 Wed 38-SEP-92 08:26

1.5

FTP Y4.22

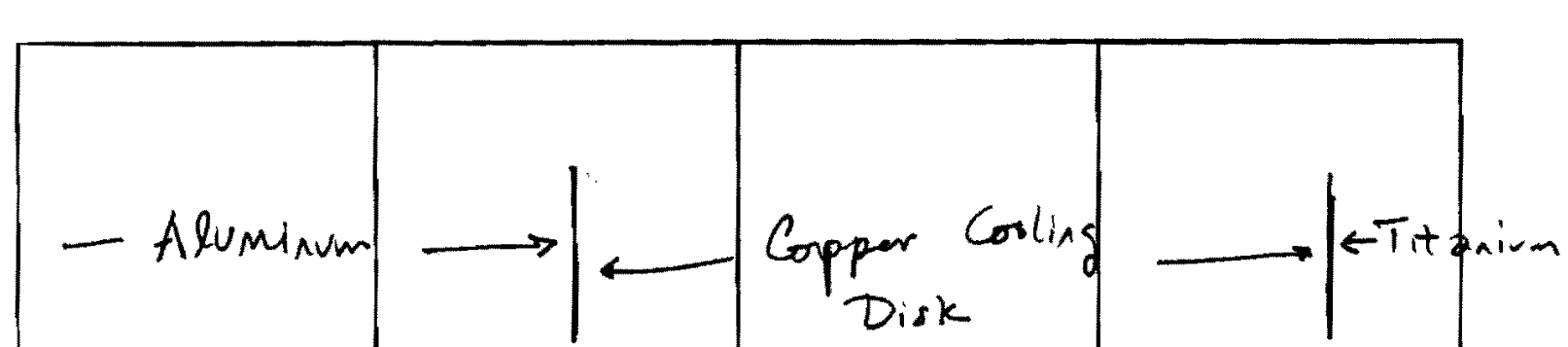

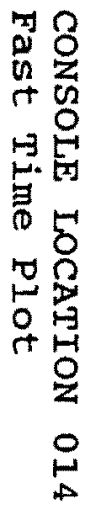

$\omega$
0
1
0
0
0
1
1
0
0
$N$
0
0
$\ddot{\omega}$
$\omega$

2.6

2.725

2.85

2.975

3.1

Hl

(Event 29) $\quad X=D:$ TRY

$A / D \quad I N C H$

engineering units

$T i$

upper cooling channel 


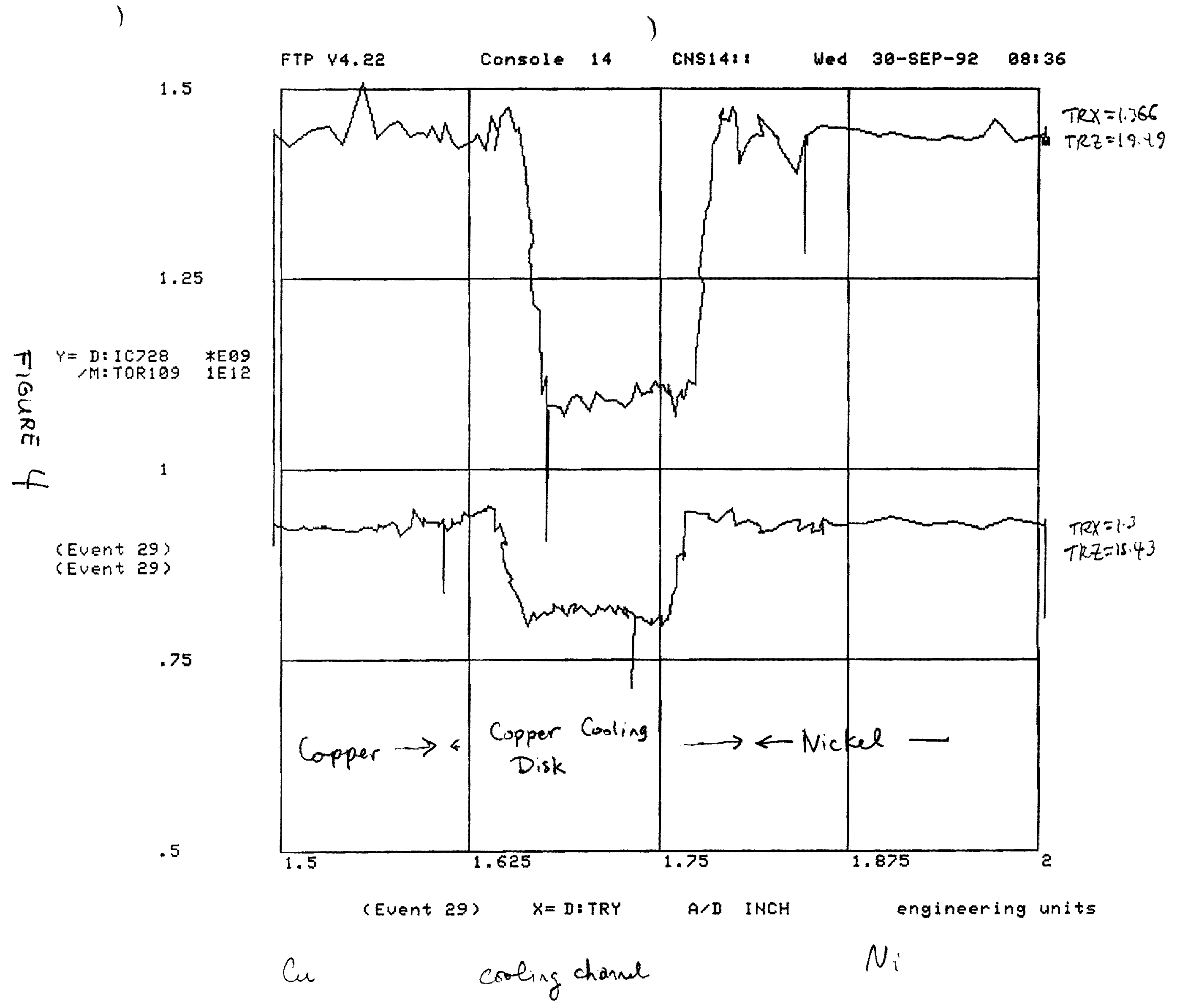




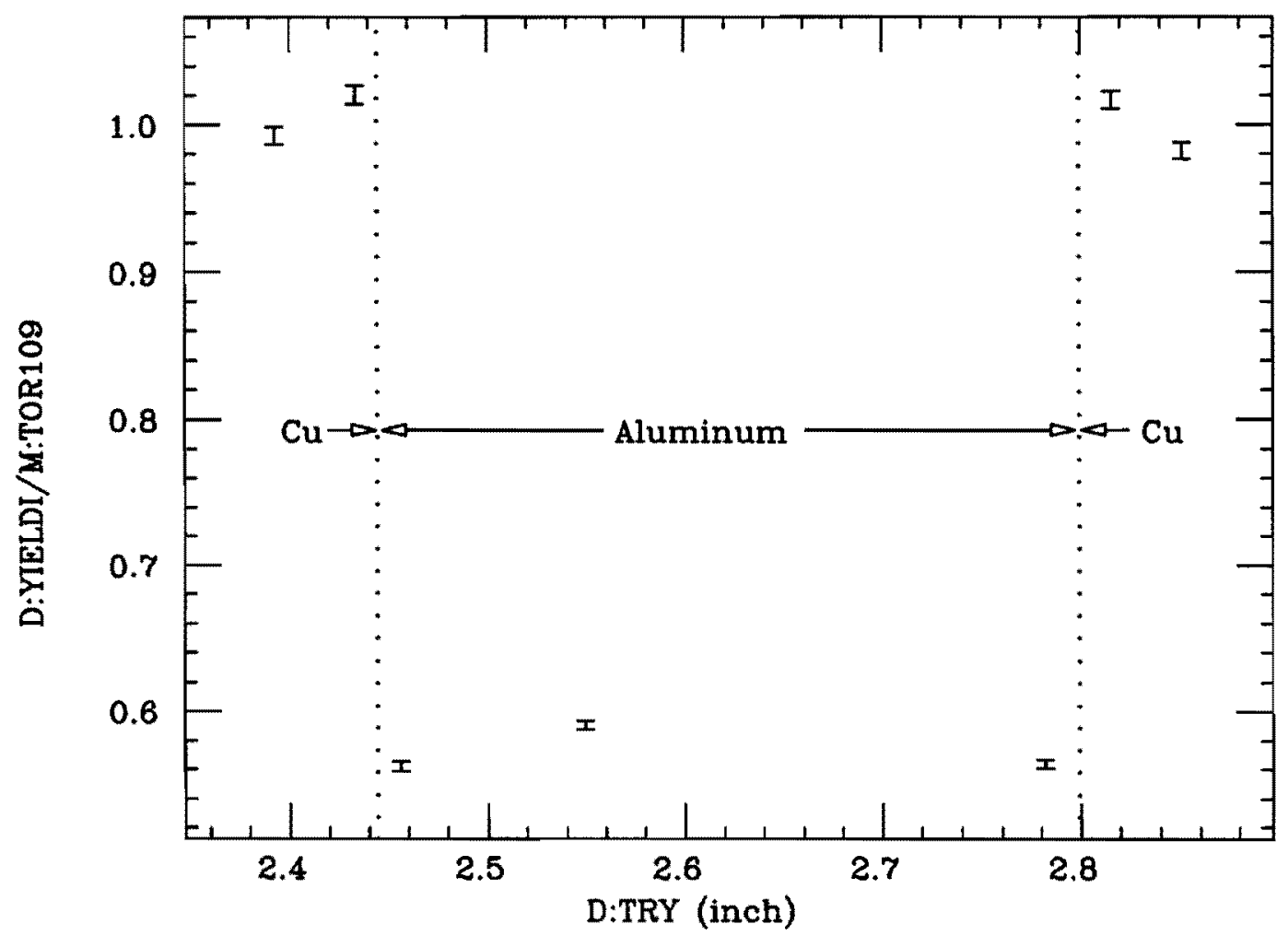

Target edge effect study: $\mathrm{Cu} / \mathrm{Al}$ interface. $\mathrm{D}: \mathrm{TRX}=1.3 ; \mathrm{D}: \mathrm{TRZ}=15.43$.

FIGURE 5 . 
Yield vs. Bunch Number(Rhenium Target)

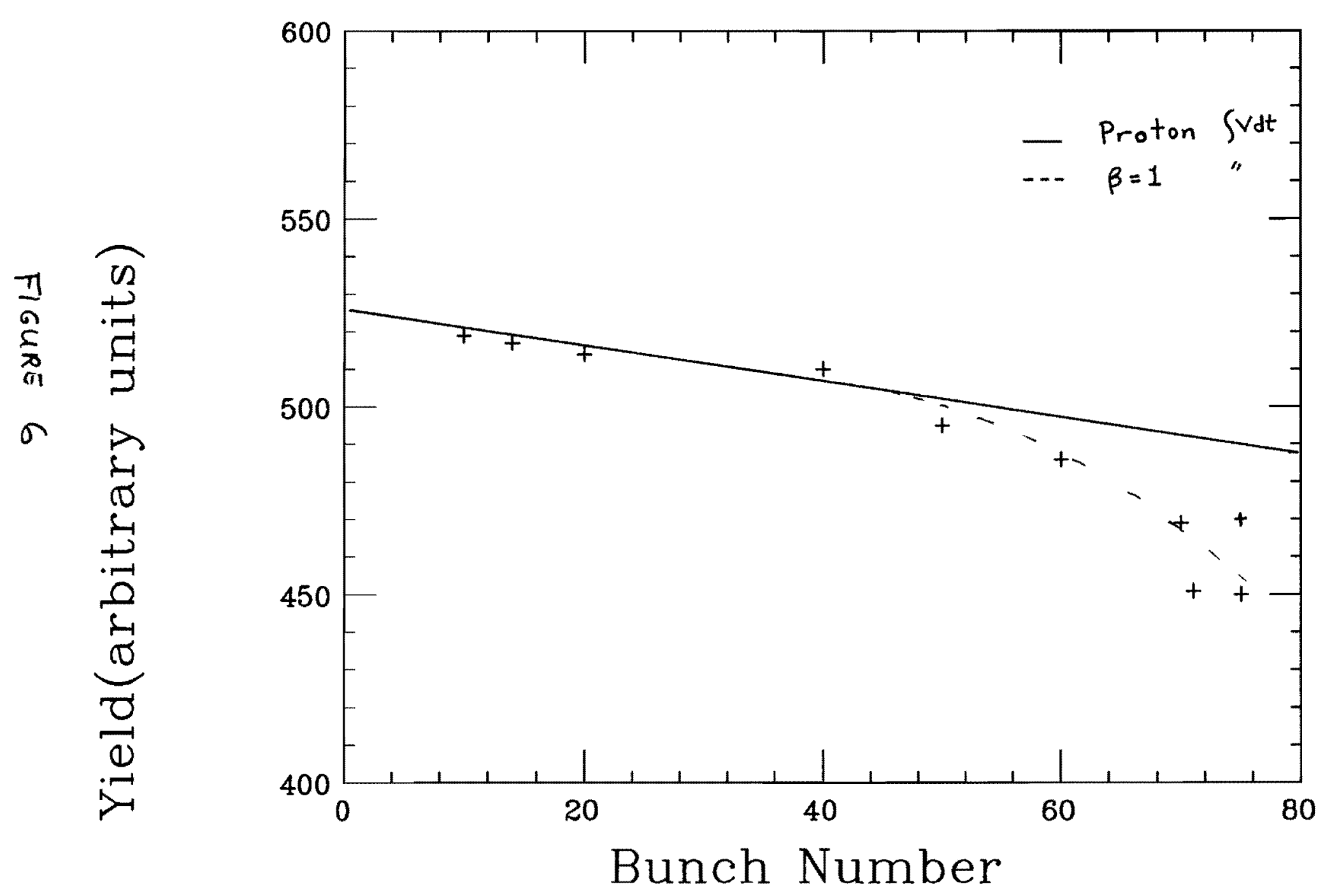




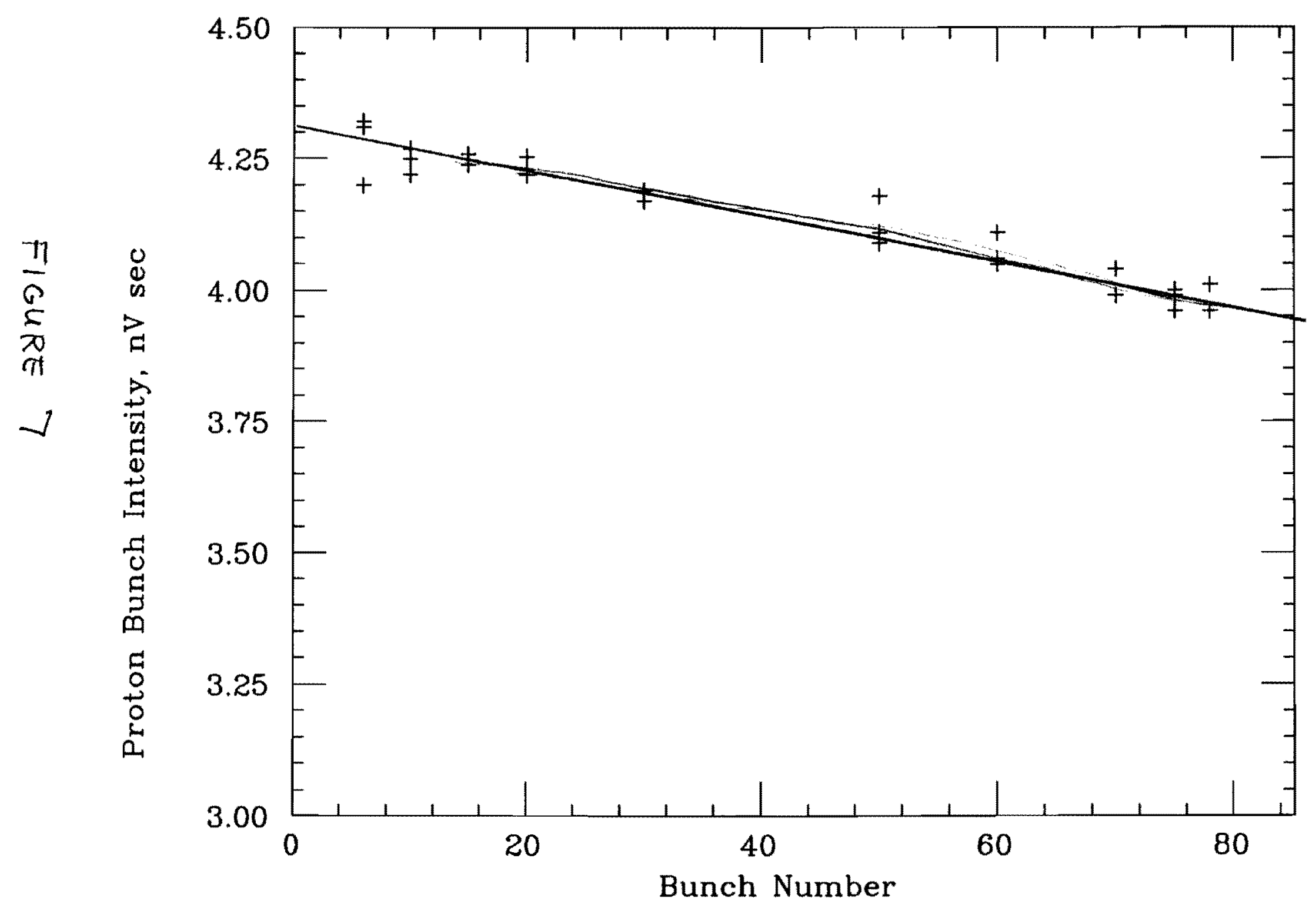




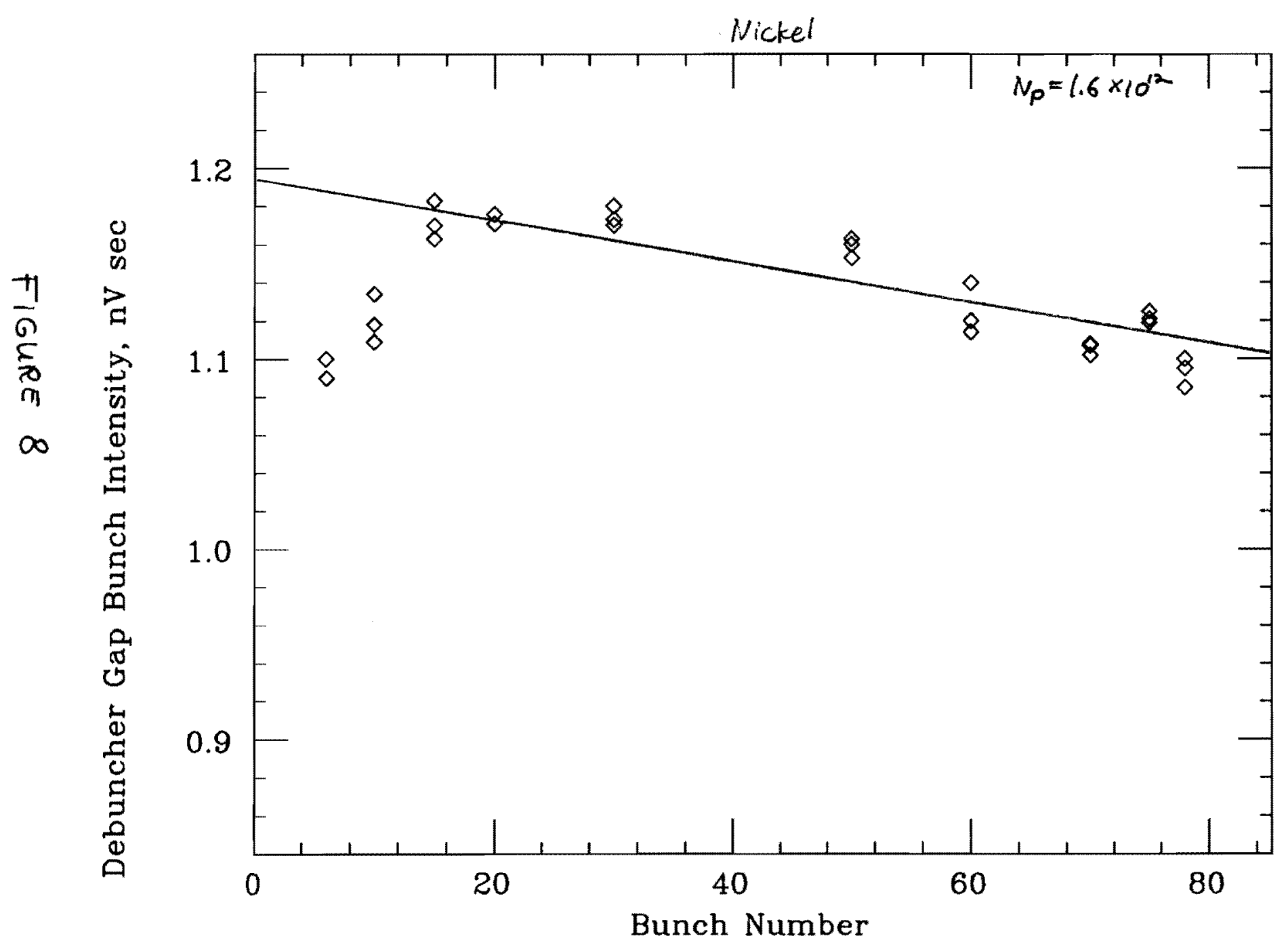

